\title{
AN AFFIRMATIVE ANSWER TO YAMADA'S CONJECTURE
}

\author{
CHUAN LIU AND FUCAI LIN
}

\begin{abstract}
In this paper, we give an affirmative answer to Yamada's Conjecture on free topological groups, which was posed in [K. Yamada, Fréchet-Urysohn spaces in free topological groups, Proc. Amer. Math. Soc., 130(2002), 2461-2469.].
\end{abstract}

\section{Introduction And Preliminaries}

Throughout this paper, all topological spaces are assumed to be Tychonoff, unless explicitly stated otherwise. Given a space $X$, let $F(X)$ and $A(X)$ be the free topological group and free Abelian topological group over $X$ in the sense of Markov respectively. For every $n \in \mathbb{N}$, let $F_{n}(X)$ and $A_{n}(X)$ denote the subspaces of $F(X)$ and $A(X)$ respectively that consists of words of reduced length at most $n$ with respect to the free basis $X$. A space $X$ is said to be Fréchet-Urysohn if, for each $x \in \bar{A} \subset X$, there exists a sequence $\left\{x_{n}\right\}$ such that $\left\{x_{n}\right\}$ converges to $x$ and $\left\{x_{n}: n \in \mathbb{N}\right\} \subset A$.

The free topological group $F(X)$ and the free abelian topological group $A(X)$ over a Tychonoff space $X$ were introduced by Markov [6] and intensively studied over the last half-century, see for example [1, 3, 4, 15, 7, , 8, 9, 10, 12.

In [1], Yamada proved the following two theorems.

Theorem 1.1. [11] Let $X$ be a metrizable space. Then $F_{5}(X)$ is Fréchet-Urysohn if and only if $X$ is discrete or compact.

Theorem 1.2. [11] Let $X$ be a metrizable space. Then $F_{3}(X)$ is Fréchet-Urysohn if and only if the set of all the non-isolated points of $X$ is compact.

Therefore, Yamada gave the following conjecture:

Yamada's Conjecture: [1] If the set of all non-isolated points of a metrizable space $X$ is compact, then $F_{4}(X)$ is Fréchet-Urysohn.

In this paper, we shall give an affirmative answer to Yamada's Conjecture.

Let $X$ be a non-empty Tychonoff space. Throughout this paper, $X^{-1}:=\left\{x^{-1}: x \in\right.$ $X$ \}, which is just a copy of $X$. For every $n \in \mathbb{N}, F_{n}(X)$ denotes the subspace of $F(X)$ that consists of all words of reduced length at most $n$ with respect to the free basis $X$. Let $e$ be the neutral element of $F(X)$ (i.e., the empty word). For every $n \in \mathbb{N}$ and an element $\left(x_{1}, x_{2}, \cdots, x_{n}\right)$ of $\left(X \bigoplus X^{-1} \bigoplus\{e\}\right)^{n}$ we call $g=x_{1} x_{2} \cdots x_{n}$ a word. This word $g$ is called reduced if it does not contain $e$ or any pair of consecutive symbols of the form $x x^{-1}$ or $x^{-1} x$. It follows that if the word $g$ is reduced and non-empty, then it is different from the neutral element $e$ of $F(X)$. In particular, each element $g \in F(X)$

2010 Mathematics Subject Classification. primary 22A30; secondary 54D10; 54E99; 54H99.

Key words and phrases. Free topological groups; Fréchet-Urysohn; compact spaces; metrizable.

The second author is supported by the NSFC (No. 11571158), the Natural Science Foundation of Fujian Province (No. 2017J01405) of China, the Program for New Century Excellent Talents in Fujian Province University, the Project for Education Reform of Fujian Education Department (No. FBJG20170182), the Institute of Meteorological Big Data-Digital Fujian and Fujian Key Laboratory of Data Science and Statistics. 
distinct from the neutral element can be uniquely written in the form $g=x_{1}^{r_{1}} x_{2}^{r_{2}} \cdots x_{n}^{r_{n}}$, where $n \geq 1, r_{i} \in \mathbb{Z} \backslash\{0\}, x_{i} \in X$, and $x_{i} \neq x_{i+1}$ for each $i=1, \cdots, n-1$, and the support of $g=x_{1}^{r_{1}} x_{2}^{r_{2}} \cdots x_{n}^{r_{n}}$ is defined as $\operatorname{supp}(g):=\left\{x_{1}, \cdots, x_{n}\right\}$. Given a subset $K$ of $F(X)$, we define $\operatorname{supp}(K):=\bigcup_{g \in K} \operatorname{supp}(g)$.

\section{The proof of Yamada's Conjecture}

Throughout this paper, we always assume that $(X, d)$ is a metric space with a metric $d$ such that the set $K$ of all the non-isolated points of $X$ is compact. For each $n \in \mathbb{N}$, let

$$
V_{n}=\{x \in X: d(x, K)<1 / n\} .
$$

Let $W_{1}=X \backslash V_{1}$ and $W_{n}=V_{n-1} \backslash V_{n}$ for each $n \geq 2$. It is easy to see that each $W_{n}$ is a closed discrete subspace.

The following lemma play an important role in our proof.

Lemma 2.1. There is a compatible metric @ on $(X, d)$, which satisfies the following conditions:

(1) $\varrho(x, y)=\varrho(y, x)$ for any $x, y \in X$;

(2) $\varrho(x, y)=|i-j| /(i \cdot j)$ if $x \in W_{i}, y \in W_{j}, i \neq j$ and $d(x, y)<|i-j| /(i \cdot j)$;

(3) $\varrho(x, y)=1 /(i \cdot(i+1))$ if $x, y \in W_{i}, x \neq y$ and $d(x, y)<1 /(i \cdot(i+1))$;

(4) $\varrho(x, y)=d(x, y)$, otherwise.

Proof. First we prove $\varrho$ is a metric on $X$. Clearly, it suffices to prove the triangle inequality. Take arbitrary $x, y, z \in X$. We may assume that $x, y, z$ are distinct each other.

Case $1|\{x, y, z\} \cap K| \geq 2$.

Then $\varrho(x, y)=d(x, y), \varrho(x, z)=d(x, z)$ and $\varrho(z, y)=d(z, y)$. Since $d(x, y) \leq d(x, z)+$ $d(z, y), \varrho(x, y) \leq \varrho(x, z)+\varrho(z, y)$.

Case $2|\{x, y, z\} \cap K|=1$.

Without loss of generality, we may assume that $x \in K$. Obviously, there exist $i, j \in \mathbb{N}$ such that $y \in W_{i}$ and $z \in W_{j}$. Then $\varrho(x, y)=d(x, y), \varrho(x, z)=d(x, z)$. It follows from the definition of $\varrho$ that $d(y, z) \leq \varrho(y, z)$. Therefore, $\varrho(x, y)=d(x, y) \leq$ $d(x, z)+d(z, y)=\varrho(x, z)+d(z, y) \leq \varrho(x, z)+\varrho(y, z) ; \varrho(x, z)=d(x, z) \leq d(x, y)+$ $d(y, z)=\varrho(x, y)+d(y, z) \leq \varrho(x, y)+\varrho(y, z)$. If $i=j$ and $d(y, z)<1 /(i \cdot(i+1))$, then $\varrho(y, z)=1 /(i \cdot(i+1))$, hence $\varrho(y, z) \leq \varrho(y, x)+\varrho(x, z)$ since $\varrho(x, y) \geq 1 / i$ and $\varrho(x, z) \geq 1 / i$. If $i=j$ and $d(y, z) \geq 1 /(i \cdot(i+1))$, then it is obvious. If $i \neq j$ and $d(y, z)<|i-j| /(i \cdot j)$, then $\varrho(y, z)=|i-j| /(i \cdot j)$, hence $\varrho(y, z) \leq \varrho(y, x)+\varrho(x, z)$ since $\varrho(x, y) \geq 1 / i$ and $\varrho(x, z) \geq 1 / j$. If $i \neq j$ and $d(y, z) \geq|i-j| /(i \cdot j)$, then it is obvious.

Case $3|\{x, y, z\} \cap K|=0$.

Then there exist $i, j, k \in \mathbb{N}$ such that $x \in W_{i}, y \in W_{j}, z \in W_{k}$. Without loss of generality, it suffices to prove $\varrho(x, y) \leq \varrho(x, z)+\varrho(y, z)$.

If $i=j$ and $d(x, y)<1 /(i \cdot(i+1))$, then $\varrho(x, y)=1 /(i \cdot(i+1))$. Moreover, $\varrho(x, z) \geq|i-k| /(i \cdot k)$ and $\varrho(y, z) \geq|i-k| /(i \cdot k)$. Assume that $i=k$, then $\varrho(x, z)+$ $\varrho(z, y)=1 /(i \cdot(i+1))+1 /(i \cdot(i+1))=2 /(i \cdot(i+1)) \geq 1 /(i \cdot(i+1))=\varrho(x, y)$. Hence it suffices to consider $i \neq k$. Then $\varrho(x, z)+\varrho(z, y) \geq|i-k| /(i \cdot k)+|i-k| /(i \cdot k)=$ $2|i-k| /(i \cdot k) \geq 1 /(i \cdot(i+1))=\varrho(x, y)$. If $i=j$ and $d(x, y) \geq 1 /(i \cdot(i+1))$, then $\varrho(x, y)=d(x, y) \leq d(x, z)+d(y, z) \leq \varrho(x, z)+\varrho(y, z)$. If $i \neq j$ and $d(x, y)<|i-j| /(i \cdot j)$, then $\varrho(x, y)=|i-j| /(i \cdot j)$, hence $\varrho(x, z)+\varrho(z, y) \geq|i-k| /(i \cdot k)+|j-k| /(j \cdot k)=$ 
$\frac{|i j-j k|+|i j-i k|}{i j k} \geq \frac{|i k-j k|}{i j k}=|i-j| /(i \cdot j)=\varrho(x, y)$. If $i \neq j$ and $d(x, y) \geq|i-j| /(i \cdot j)$, then $\varrho(x, y)=d(x, y) \leq d(x, z)+d(y, z) \leq \varrho(x, z)+\varrho(y, z)$.

Therefore, $\varrho$ is a metric. It easily check that the topology generated by the metric $\varrho$ on $X$ is compatible with $(X, d)$.

Lemma 2.2. The metric @ in Lemma 2.1 has the following properties:

(1) For any $x \in X \backslash V_{k}, y \in X, y \neq x$, it has $\varrho(x, y)>1 /(k+1)^{2}$.

(2) If $\varrho\left(x_{n}, y_{n}\right)<1 / n$ for each $n \in \mathbb{N}$, then there exist sequences $\left\{x_{n_{k}}\right\}$ and $\left\{y_{n_{k}}\right\}$ such that $x_{n_{k}} \rightarrow x_{0}$ and $y_{n_{k}} \rightarrow x_{0}$ as $k \rightarrow \infty$, where $x_{n}, y_{n} \in X, x_{n} \neq y_{n}$ and $x_{0} \in K$.

Let $\varrho^{*}$ and $N_{\varrho}$ be defined in the proof in [2, Theorem 7.2.2]. For the convenience, we give out the definitions.

Suppose that $e$ is the neutral element of the abstract free group $F_{a}(X)$ on $X$. Extend $\varrho$ from $X$ to a metric $\varrho^{*}$ on $X \cup\{e\} \cup X^{-1}$. Choose a point $x_{0} \in X$ and for every $x \in X$, put

$$
\varrho^{*}(e, x)=\varrho^{*}\left(e, x^{-1}\right)=1+\varrho\left(x_{0}, x\right) .
$$

Then for $x, y \in X$, define the distance $\varrho^{*}\left(x^{-1}, y^{-1}\right), \varrho^{*}\left(x^{-1}, y\right)$ and $\varrho^{*}\left(x, y^{-1}\right)$ by

$$
\begin{gathered}
\varrho^{*}\left(x^{-1}, y^{-1}\right)=\varrho^{*}(x, y)=\varrho(x, y), \\
\varrho^{*}\left(x^{-1}, y\right)=\varrho^{*}\left(x, y^{-1}\right)=\varrho^{*}(x, e)+\varrho^{*}(e, y) .
\end{gathered}
$$

Let $A$ be a subset of $\mathbb{N}$ such that $|A|=2 n$ for some $n \geq 1$. A scheme on $A$ is a partition of $A$ to pairs $\left\{a_{i}, b_{i}\right\}$ with $a_{i}<b_{i}$ such that each two intervals $\left[a_{i}, b_{i}\right]$ and $\left[a_{j}, b_{j}\right]$ in $\mathbb{N}$ are either disjoint or one contains the other.

If $\mathcal{X}$ is a word in the alphabet $X \cup\{e\} \cup X^{-1}$, then we denote the reduced form and the length of $\mathcal{X}$ by $[\mathcal{X}]$ and $\ell(\mathcal{X})$ respectively.

For each $n \in \mathbb{N}$, let $\mathcal{S}_{n}$ be the family of all schemes $\varphi$ on $\{1,2, \cdots, 2 n\}$. As in [2], define

$$
\Gamma_{\varrho}(\mathcal{X}, \varphi)=\frac{1}{2} \sum_{i=1}^{2 n} \varrho^{*}\left(x_{i}^{-1}, x_{\varphi(i)}\right) .
$$

Then we define a prenorm $N_{\varrho}: F_{a}(X) \rightarrow[0,+\infty)$ by setting $N_{\varrho}(g)=0$ if $g=e$ and

$$
N_{\varrho}(g)=\inf \left\{\Gamma_{\varrho}(\mathcal{X}, \varphi):[\mathcal{X}]=g, \ell(\mathcal{X})=2 n, \varphi \in \mathcal{S}_{n}, n \in \mathbb{N}\right\}
$$

if $g \in F_{a}(X) \backslash\{e\}$.

Lemma 2.3. Let

$$
B=\left\{x^{\epsilon_{1}} y^{\epsilon_{2}} z^{\epsilon_{3}} t^{\epsilon_{4}} \in F_{4}(X) \backslash F_{3}(X): x, y, z, t \in X, \epsilon_{i} \in\{-1,1\}, \sum \epsilon_{i}=0,1 \leq i \leq 4\right\} .
$$

If $e \in \bar{B}$, then there is a convergent sequence $\left\{h_{n}=x_{n}^{\epsilon_{1}} y_{n}^{\epsilon_{2}} z_{n}^{\epsilon_{3}} t_{n}^{\epsilon_{4}}\right\}$ in $B$ such that $h_{n} \rightarrow e$ as $n \rightarrow \infty$.

Proof. Let $\varrho^{*}$ and $N_{\varrho}$ be defined as above. It is known that for each $n \in \mathbb{N}$,

$$
U_{\varrho}(n)=\left\{g \in F_{a}(X): N_{\varrho}(g)<1 / n\right\} \cap F_{0}(X)
$$

is an open neighborhood of $e$ in $F(X)$ by [2, Theorem 7.2.2]. We divide the proof into the following two cases.

Case 1: For each $n \in \mathbb{N}$, there exists $x_{n}^{\epsilon_{1}(n)} y_{n}^{\epsilon_{2}(n)} z_{n}^{\epsilon_{3}(n)} t_{n}^{\epsilon_{4}(n)} \in B \cap U_{\varrho}(n)$ such that $x_{n}, y_{n}, z_{n}, t_{n} \in V_{n}$, where $V_{n}=\{x \in X: d(x, K)<1 / n\}$.

Without loss of generality, we may assume that $\epsilon_{i}(n)=\epsilon_{i}(i \leq 4)$. In fact, we can choose a subsequence of $\left\{x_{n}^{\epsilon_{1}(n)} y_{n}^{\epsilon_{2}(n)} z_{n}^{\epsilon_{3}(n)} t_{n}^{\epsilon_{4}(n)}: n \in \mathbb{N}\right\}$. We can also assume that 
$x_{n} \rightarrow x, y_{n} \rightarrow y, z_{n} \rightarrow z, t_{n} \rightarrow t$, where $x, y, z, t \in K$. Clearly, $\sum \epsilon_{i}=0$. Then we have the following claim.

Claim 1: $x^{\epsilon_{1}} y^{\epsilon_{2}} z^{\epsilon_{3}} t^{\epsilon_{4}}=e$.

Fix an $n \in \mathbb{N}$, let $g_{n}=x_{n}^{\epsilon_{1}} y_{n}^{\epsilon_{2}} z_{n}^{\epsilon_{3}} t_{n}^{\epsilon_{4}}$ and let $D_{n}=\left\{x_{n}^{\epsilon_{1}}, y_{n}^{\epsilon_{2}}, z_{n}^{\epsilon_{3}}, t_{n}^{\epsilon_{4}}\right\}$. We claim that

$$
N_{\varrho}\left(g_{n}\right)=\min \left\{\varrho^{*}\left(x_{n}^{-\epsilon_{1}}, y_{n}^{\epsilon_{2}}\right)+\varrho^{*}\left(z_{n}^{-\epsilon_{3}}, t_{n}^{\epsilon_{4}}\right), \varrho^{*}\left(x_{n}^{-\epsilon_{1}}, t_{n}^{\epsilon_{4}}\right)+\varrho^{*}\left(y_{n}^{-\epsilon_{3}}, z_{n}^{\epsilon_{4}}\right)\right\}
$$

if $N_{\varrho}\left(g_{n}\right)<\frac{1}{n}$.

In fact, it follows from Claim 1 of the proof in [2, Theorem 7.2.2] that there exist an almost reduced word $\Upsilon_{g_{n}}=p_{1} \cdots p_{2 m}$ with $2 m \leq 8$ and a scheme $\varphi_{g_{n}}$ such that $\Upsilon_{g_{n}}$ contains only the letters of $g_{n}$ or the letter $e$ and $N_{\varrho}\left(g_{n}\right)=\Gamma_{\varrho}\left(\Upsilon_{g_{n}}, \varphi_{g_{n}}\right)$, where $\Gamma_{\varrho}\left(\Upsilon_{g_{n}}, \varphi_{g_{n}}\right)=\frac{1}{2} \sum_{i=1}^{2 m} \varrho^{*}\left(p_{i}^{-1}, p_{\varphi_{g_{n}}(i)}\right)$. If $p_{i} \in D_{n}$, then $p_{\varphi_{g_{n}}(i)} \in D_{n}$; otherwise, $p_{\varphi_{g_{n}}(i)}=e$, then $\Gamma_{\varrho}\left(\Upsilon_{g_{n}}, \varphi_{g_{n}}\right) \geq 1$, which is a contradiction with $N_{\varrho}\left(g_{n}\right)<\frac{1}{n}$.

Subcase 1.1: $\varrho^{*}\left(x_{n}^{-\epsilon_{1}}, y_{n}^{\epsilon_{2}}\right)+\varrho^{*}\left(z_{n}^{-\epsilon_{3}}, t_{n}^{\epsilon_{4}}\right)<1 / n$ for infinitely many $n \in \mathbb{N}$.

Then $\epsilon_{1}=-\epsilon_{2}$ and $\epsilon_{3}=-\epsilon_{4}$. If $x \neq y$, then $\varrho(x, y)=r>0$. It is easy to see that there is $k \in \mathbb{N}$ such that $\varrho\left(x, x_{i}\right)<r / 3, \varrho\left(x_{i}, y_{i}\right)<r / 3, \varrho\left(y_{i}, y\right)<r / 3$ whenever $i>k$. Then $r=\varrho(x, y) \leq \varrho\left(x, x_{i}\right)+\varrho\left(x_{i}, y_{i}\right)+\varrho\left(y_{i}, y\right)<r / 3+r / 3+r / 3=r$. This is a contradiction, hence $x=y$. Similarly, $z=t$. Therefore $x^{\epsilon_{1}} y^{\epsilon_{2}} z^{\epsilon_{3}} t^{\epsilon_{4}}=e$.

Subcase 1.2: $\varrho^{*}\left(x_{n}^{-\epsilon_{1}}, t_{n}^{\epsilon_{4}}\right)+\varrho^{*}\left(y_{n}^{-\epsilon_{3}}, z_{n}^{\epsilon_{4}}\right)<1 / n$ for infinitely many $n \in \mathbb{N}$.

Then $\epsilon_{1}=-\epsilon_{4}, \epsilon_{2}=-\epsilon_{3}$. By the proof of Subcase 1.1, we can prove that $x=t, y=z$. Then $x^{\epsilon_{1}} y^{\epsilon_{2}} z^{\epsilon_{3}} t^{\epsilon_{4}}=e$. The proof of Claim 1 is completed.

By Claim 1, we see that Lemma 2.3 holds.

Case 2: There is $n \in \mathbb{N}$ such that for any $a^{\epsilon_{1}} b^{\epsilon_{2}} c^{\epsilon_{3}} d^{\epsilon_{4}} \in U_{\varrho}(n) \cap B=A$, one of $a, b, c, d$ is not in $V_{n}$.

We only consider the case

$$
e \in \overline{\left\{a^{\epsilon_{1}} b^{\epsilon_{2}} c^{\epsilon_{3}} d^{\epsilon_{4}} \in A: a \notin V_{n}\right\} \cup\left\{a^{\epsilon_{1}} b^{\epsilon_{2}} c^{\epsilon_{3}} d^{\epsilon_{4}} \in A: b \notin V_{n}\right\}},
$$

otherwise consider the set $\left(U_{\varrho}(n) \cap B\right)^{-1}$. First, we prove that $e \in \overline{\left\{a^{\epsilon_{1}} b^{\epsilon_{2}} c^{\epsilon_{3}} d^{\epsilon_{4}} \in A: a \notin V_{n}\right\}}$, then it suffices to prove

$$
e \notin \overline{\left\{a^{\epsilon_{1}} b^{\epsilon_{2}} c^{\epsilon_{3}} d^{\epsilon_{4}} \in A: b \notin V_{n}\right\}} .
$$

Let

$$
A_{1}=\left\{a^{\epsilon_{1}} b^{\epsilon_{2}} c^{\epsilon_{3}} d^{\epsilon_{4}} \in A: b \notin V_{n}\right\} .
$$

Assume that $e \in \overline{A_{1}}$. In order to obtain a contradiction, we find a neighborhood $W_{1}$ of $e$ such that $W_{1} \cap A_{1}=\emptyset$. Indeed, let

$$
W_{1}=\left\{g \in F(X): N_{\varrho}(g)<1 /(n+1)^{2}\right\} .
$$

Then $W_{1}$ is an open neighborhood of $e$. Moreover, for any $g=a^{\epsilon_{1}} b^{\epsilon_{2}} c^{\epsilon_{3}} d^{\epsilon_{4}} \in A_{1}$, we prove that $N_{\varrho}(g) \geq 1 /(n+1)^{2}$. Indeed, it follows from Claim 1 of the proof of [2, Theorem 7.2.2] that there exist an almost reduced word $\Upsilon_{g}=x_{1} \cdots x_{2 m}$ and a scheme $\varphi_{g}$ such that satisfies the following conditions:

(i) $\Upsilon_{g}$ contains only the letters of $g$ or the letter $e$;

(ii) $\left[\Upsilon_{g}\right]=g$ and $\ell\left(\Upsilon_{g}\right) \leq 2 \ell(g)$;

(iii) $N_{\varrho}(g)=\Gamma_{\varrho}\left(\Upsilon_{g}, \varphi_{g}\right)$.

We claim that $\Gamma_{\varrho}\left(\Upsilon_{g}, \varphi_{g}\right) \geq \frac{1}{(n+1)^{2}}$. Indeed, it follows from (i) and (ii) that there exists $i_{0} \leq 2 m$ such that $x_{i_{0}}=b^{\epsilon_{2}}$, then $\varrho^{*}\left(x_{i_{0}}^{-1}, x_{\varphi_{g}\left(i_{0}\right)}\right)=\varrho^{*}\left(b^{-\epsilon_{2}}, x_{\varphi_{g}\left(i_{0}\right)}\right)$. Then we can complete the proof by the following (a)-(d).

(a) If $x_{\varphi_{g}\left(i_{0}\right)}=e$, then $\varrho^{*}\left(b^{-\epsilon_{2}}, x_{\varphi_{g}\left(i_{0}\right)}\right) \geq 1$. 
(b) If $x_{\varphi_{g}\left(i_{0}\right)}=a^{\epsilon_{1}}$, then it follows from (1) of Lemma 2.2 that $\varrho^{*}\left(b^{-\epsilon_{2}}, x_{\varphi_{g}\left(i_{0}\right)}\right)=$ $\varrho^{*}\left(b^{-\epsilon_{2}}, a^{\epsilon_{1}}\right)>\frac{1}{(n+1)^{2}}$ since $a^{\epsilon_{1}} b^{\epsilon_{2}} \neq e$ and $b \notin V_{n}$.

(c) Assume $x_{\varphi_{g}\left(i_{0}\right)}=c^{\epsilon_{3}}$. If $\epsilon_{2}=\epsilon_{3}$ and $b=c$, then $x_{\varphi_{g}\left(i_{0}\right)}=b^{\epsilon_{2}}$, hence $\varrho^{*}\left(b^{-\epsilon_{2}}, x_{\varphi_{g}\left(i_{0}\right)}\right)=$ $\varrho^{*}\left(b^{-\epsilon_{2}}, b^{\epsilon_{2}}\right) \geq 1 \geq \frac{1}{(n+1)^{2}}$. If $b \neq c$, then it follows from (1) of Lemma 2.2 that $\varrho^{*}\left(b^{-\epsilon_{2}}, x_{\varphi_{g}\left(i_{0}\right)}\right)=\varrho^{*}\left(b^{-\epsilon_{2}}, c^{\epsilon_{3}}\right)>\frac{1}{(n+1)^{2}}$ since $b \neq c$ and $b \notin V_{n}$.

(d) Assume $x_{\varphi_{g}\left(i_{0}\right)}=d^{\epsilon_{4}}$. Obviously, there exists a $j_{0} \leq 2 m$ such that $x_{j_{0}}=c^{\epsilon_{3}}$. Since $\varphi_{g}$ is a scheme, it has $x_{\varphi_{g}\left(j_{0}\right)}=e$, hence $\varrho^{*}\left(x_{j_{0}}^{-1}, x_{\varphi_{g}\left(j_{0}\right)}\right) \geq 1$. Then

$$
\Gamma_{\varrho}\left(\Upsilon_{g}, \varphi_{g}\right) \geq \varrho^{*}\left(b^{-\epsilon_{2}}, x_{\varphi_{g}\left(i_{0}\right)}\right)+\varrho^{*}\left(x_{j_{0}}^{-1}, x_{\varphi_{g}\left(j_{0}\right)}\right) \geq 1 \geq \frac{1}{(n+1)^{2}} .
$$

Therefore, $W_{1} \cap A_{1}=\emptyset$, which is a contradiction. Hence the point $e$ belongs to the closure of $\left\{a^{\epsilon_{1}} b^{\epsilon_{2}} c^{\epsilon_{3}} d^{\epsilon_{4}} \in A: a \notin V_{n}\right\}$. Further, we claim that $e$ does not belong to the closure of the set

$$
A_{2}=\left\{a^{\epsilon_{1}} b^{\epsilon_{2}} c^{\epsilon_{3}} d^{\epsilon_{4}} \in A: a \neq d, a \notin V_{n}\right\} \cup\left\{a^{\epsilon_{1}} b^{\epsilon_{2}} c^{\epsilon_{3}} d^{\epsilon_{4}} \in A: a=d, \epsilon_{1}=\epsilon_{4}, a \notin V_{n}\right\} .
$$

Suppose not, assume that $e \in \overline{A_{2}}$. In order to obtain a contradiction, it suffices to prove that $W_{1} \cap A_{2}=\emptyset$ by a similar proof above.

Therefore, $e$ belongs to the closure of $A_{3}=\left\{a^{\epsilon_{1}} b^{\epsilon_{2}} c^{-\epsilon_{2}} a^{-\epsilon_{1}} \in A: a \notin V_{n}\right\}$. Next we prove that there exists a convergent sequence in $A_{3}$ which converges to $e$.

Let $D=\left\{a \in X: a^{\epsilon_{1}} b^{\epsilon_{2}} c^{-\epsilon_{2}} a^{-\epsilon_{1}} \in A_{3}\right\}$, and let $A_{a}=\left\{a^{\epsilon_{1}} b^{\epsilon_{2}} c^{-\epsilon_{2}} a^{-\epsilon_{1}} \in A_{3}\right\}$ for each $a \in D$. It is obvious that $A_{3}=\bigcup_{a \in D} A_{a}$. We claim that there exist $a \in D$, an infinite subset $M$ of $\mathbb{N}, b_{i} \in V_{i}$ and $c_{i} \in V_{i}$ for any $i \in M$ such that

$$
a^{\epsilon_{1}} b_{i}^{\epsilon_{2}} c_{i}^{-\epsilon_{2}} a^{-\epsilon_{1}} \in A_{3} \cap U_{\varrho}(i) .
$$

Suppose not, for each $a \in D$, there is $n_{a} \in \mathbb{N}\left(n_{\alpha}>n\right)$ such that either $b \notin V_{n_{a}}$ or $c \notin V_{n_{a}}$ for each $a^{\epsilon_{1}} b^{\epsilon_{2}} c^{-\epsilon_{2}} a^{-\epsilon_{1}} \in A_{a}$. Without loss of generality, we may assume that $e$ belongs to the closure of $A_{4}=\left\{a^{\epsilon_{1}} b^{\epsilon_{2}} c^{-\epsilon_{2}} a^{-\epsilon_{1}} \in A_{3}: a \in D, b \notin V_{n_{a}}, c \in V_{n}\right\}$. Let $C^{\prime}=\left\{b, c \in X: a^{\epsilon_{1}} b^{\epsilon_{2}} c^{-\epsilon_{2}} a^{-\epsilon_{1}} \in A_{4}\right\}$ and $C=\overline{C^{\prime}}$. Obviously, $C \cap D=\emptyset, D$ is closed discrete and $C$ is closed in $X$. Define a mapping $f=\pi \circ \bar{\psi}: F(C \oplus D) \rightarrow A(C \times F(D))$, where the mappings $\pi$ and $\bar{\psi}$ are defined in the proof of [11, Theorem 2.4]. By our definition of $C$ and $D$, it is easy to see that $\pi, \bar{\psi}$ and $f$ are all continuous. Therefore, $0=f(e) \in \overline{f\left(A_{4}\right)}$. For each $a^{\epsilon_{1}} b^{\epsilon_{2}} c^{-\epsilon_{2}} a^{-\epsilon_{1}} \in A_{4}$, we have

$$
\begin{aligned}
\bar{\psi}\left(a^{\epsilon_{1}} b^{\epsilon_{2}} c^{-\epsilon_{2}} a^{-\epsilon_{1}}\right) & =\bar{\psi}\left(a^{\epsilon_{1}}\right) \bar{\psi}\left(b^{\epsilon_{2}}\right) \bar{\psi}\left(c^{-\epsilon_{2}}\right) \bar{\psi}\left(a^{-\epsilon_{1}}\right) \\
& =\psi(a)^{\epsilon_{1}} \psi(b)^{\epsilon_{2}} \psi(c)^{-\epsilon_{2}} \psi(a)^{-\epsilon_{1}} \\
& =\left(a^{\epsilon_{1}}, 0\right)\left(e, \epsilon_{2}(b, e)\right)\left(e,-\epsilon_{2}(c, e)\right)\left(a^{-\epsilon_{1}}, 0\right) \\
& =\left(a^{\epsilon_{1}}, \epsilon_{2}\left(b, a^{\epsilon_{1}}\right)\right)\left(a^{-\epsilon_{1}},-\epsilon_{2}(c, e)\right) \\
& =\left(e, \epsilon_{2}\left(b, a^{\epsilon_{1}}\right)-\epsilon_{2}\left(c, a^{\epsilon_{1}}\right)\right),
\end{aligned}
$$

hence $f\left(a^{\epsilon_{1}} b^{\epsilon_{2}} c^{-\epsilon_{2}} a^{-\epsilon_{1}}\right)=\pi \circ \bar{\psi}\left(a^{\epsilon_{1}} b^{\epsilon_{2}} c^{-\epsilon_{2}} a^{-\epsilon_{1}}\right)=\epsilon_{2}\left(b, a^{\epsilon_{1}}\right)-\epsilon_{2}\left(c, a^{\epsilon_{1}}\right) \in A_{2}(C \times F(D))$. By the arbitrary, it follows that $\overline{f\left(A_{4}\right)} \subset A_{2}(C \times F(D))$. Since $C \times F(D)$ is a metrizable space, it follows from [10, Proposition 4.8] that $A_{2}(C \times F(D))$ is a Fréchet-Urysohn space. Then there exists a sequence $\mathcal{S}=\left\{\epsilon_{2}(k)\left(b_{k}, a_{k}^{\epsilon_{1}(k)}\right)-\epsilon_{2}(k)\left(c_{k}, a_{k}^{\epsilon_{1}(k)}\right): k \in \mathbb{N}\right\}$ which converges to 0 , where $\epsilon_{1}(k), \epsilon_{2}(k) \in\{1,-1\}$ for each $k \in \mathbb{N}$. Then $F=\overline{\operatorname{supp}(\mathcal{S} \cup\{0\})}$ is a compact subset in $C \times F(D)$. Since the projective mappings $\pi_{1}$ and $\pi_{2}$ are continuous from $C \times F(D)$ to $C$ and $F(D)$ respectively, the sets $\pi_{1}(F)$ and $\pi_{2}(F)$ are compact in $C$ and $F(D)$ respectively. Then the set $F_{1}=\left\{a_{k}^{\epsilon_{1}(k)}: k \in \mathbb{N}\right\}$ is a finite set since $F(D)$ is 
discrete. Therefore, the set $F_{2}=\left\{b_{k}: k \in \mathbb{N}\right\}$ is also finite since $\left\{b_{k}: k \in \mathbb{N}\right\} \cap V_{n_{0}}=\emptyset$, where $n_{0}=\max \left\{n_{a}: a \in F_{1}\right\}$. Therefore, there exist $a \in F_{1}$ and $b \in F_{2}$ such that some subsequence $\left\{\epsilon_{2}\left(n_{k}\right)(b, a)-\epsilon_{2}\left(n_{k}\right)\left(c_{n_{k}}, a\right): k \in \mathbb{N}\right\}$ of $\mathcal{S}$ converges to 0 . Without loss of generality, we may assume that $(b, a)-\left(c_{n_{k}}, a\right) \rightarrow 0$ as $k \rightarrow \infty$, that is, $\left(c_{n_{k}}, a\right) \rightarrow(b, a)$ as $k \rightarrow \infty$. Then $c_{n_{k}}=b$ for any sufficiently large $k$, which is a contradiction.

Then $a^{\epsilon_{1}} b_{i}^{\epsilon_{2}} c_{i}^{-\epsilon_{2}} a^{-\epsilon_{1}} \rightarrow a^{\epsilon_{1}} b^{\epsilon_{2}} c^{-\epsilon_{2}} a^{-\epsilon_{1}}$ as $i \rightarrow \infty$, where $b, c \in K$. In viewing of the proof of Subcase 1.1, we could prove that $b=c$, hence $a^{\epsilon_{1}} b_{i}^{\epsilon_{2}} c_{i}^{-\epsilon_{2}} a^{-\epsilon_{1}} \rightarrow a^{\epsilon_{1}} b^{\epsilon_{2}} b^{-\epsilon_{2}} a^{-\epsilon_{1}}=$ $e$ as $i \rightarrow \infty$.

For each $g=a_{1} \ldots a_{n} \in F_{n}(X) \backslash F_{n-1}(X)$ with $a_{1}, \ldots, a_{n} \in X \cup X^{-1}$, denote by $\mathcal{P}_{X}(g)$ the subfamily of $P(X)$ consisting of all $\varrho$ such that $\varrho^{*}\left(a_{i}^{-1}, a_{i+1}\right) \geq 1$ for each $i<n$. For each $\varrho \in \mathcal{P}_{X}(g)$, put $U_{\varrho}(g)=\left\{x_{1} \ldots x_{i} y^{\epsilon} z^{-\epsilon} x_{i+1} \ldots x_{n}: x_{1}, \ldots, x_{n} \in X \cup X^{-1}, y, z \in\right.$ $X, \epsilon= \pm 1,0 \leq i \leq n$, and $\left.\varrho(y, z)+\sum_{k=1}^{n} \varrho\left(a_{k}, x_{k}\right)<1\right\}$.

Lemma 2.4. [2, Theorem 7.2.11]. The family $\left\{U_{\varrho}(g): \varrho \in \mathcal{P}_{X}(g)\right\}$ is an open base for $F_{n+2}(X)$ at any point $g \in F_{n}(X) \backslash F_{n-1}(X)$.

Lemma 2.5. Let $B \subset F_{4}(X) \backslash F_{3}(X)$. If $g=a_{1} a_{2} \in \bar{B}$ is a reduced form, where $a_{1}, a_{2} \in X \cup X^{-1}$, then there is a convergent sequence $\left\{h_{n}=x_{n}^{\epsilon_{1}} y_{n}^{\epsilon_{2}} z_{n}^{\epsilon_{3}} t_{n}^{\epsilon_{4}}: h_{n} \in B\right\}$ such that $h_{n} \rightarrow g$ as $n \rightarrow \infty$.

Proof. Let $\varrho$ be the metric on $X$ in Lemma 2.1. If $\varrho^{*}\left(a_{1}^{-1}, a_{2}\right) \geq 1$, then $\varrho \in \mathcal{P}_{X}(g)$; if $\varrho^{*}\left(a_{1}^{-1}, a_{2}\right)=r<1$, then $(1 / r) \varrho \in \mathcal{P}_{X}(g)$. We may find a $k \in \mathbb{N}$ such that $k>1 / r$, if $r \geq 1$, then $k=1$.

Let $\varrho_{n}=n k \cdot \varrho$ for each $n \in \mathbb{N}$. By Lemma 2.4. $U_{\varrho_{n}}(g) \cap B \neq \emptyset$ for each $n \in \mathbb{N}$, thus without loss of generality it can take an arbitrary point $x_{1}(n) y^{\epsilon}(n) z^{-\epsilon}(n) x_{2}(n) \in$ $U_{\varrho_{n}}(g) \cap B$. Then $n \cdot \varrho(y(n), z(n))<1, n \cdot \varrho\left(x_{1}(n), a_{1}\right)<1$ and $n \cdot \varrho\left(x_{2}(n), a_{2}\right)<1$. By Lemma 2.2. $y(n) \rightarrow f, z(n) \rightarrow f, x_{i}(n) \rightarrow a_{i}(i \leq 2)$ as $n \rightarrow \infty$. Therefore, $x_{1}(n) y^{\epsilon}(n) z^{-\epsilon}(n) x_{2}(n) \rightarrow a_{1} f f^{-1} a_{2}=a_{1} a_{2}=g$.

Now, we can give an affirmative answer to Yamada's Conjecture1 1 .

Theorem 2.6. Let $X$ be a metrizable space in which the set of all the non-isolated points is compact. Then $F_{4}(X)$ is Fréchet-Urysohn.

Proof. Assume that $g \in \bar{B}$, where $B \subset F_{4}(X)$ and $g \in F_{4}(X)$. Next we prove that there exists a sequence in $B$ which converges to $g$. It is well-known that

$F_{4}(X)=\left[\left(F_{4}(X) \backslash F_{3}(X)\right) \cup\left(F_{2}(X) \backslash F_{1}(X)\right) \cup\{e\}\right] \oplus\left[\left(F_{3}(X) \backslash F_{2}(X)\right) \cup\left(F_{1}(X) \backslash\{e\}\right)\right]$.

If $g \in \overline{F_{3}(X) \cap B}$, we can find a sequence in $B$ converging to $g$ since $F_{3}(X)$ is FréchetUrysohn by Theorem 1.2 .

If $g \in \overline{\left(F_{4}(X) \backslash F_{3}(X)\right) \cap B}$, we consider the following cases:

(a) $g \in F_{4}(X) \backslash F_{3}(X)$. By [2, Theorem 7.6.2], $F_{4}(X) \backslash F_{3}(X)$ is metrizable, we can find a sequence in $B$ converges to $g$.

(b) $g \in F_{2}(X) \backslash F_{1}(X)$. By Lemma 2.5, we can find a sequence in $B$ converges to $g$.

(c) $g=e$. By Lemma 2.3, there is a sequence in $B$ converging to $e$.

Therefore $F_{4}(X)$ is Fréchet-Urysohn.

Acknowledgements. The authors are thankful to the referee for valuable remarks and corrections and all other sort of help related to the content of this article.

\footnotetext{
${ }^{1}$ Professor K. Yamada informed us that he just proved this conjecture independently.
} 


\section{REFERENCES}

[1] A.V. Arhangel'skiǐ, O.G. Okunev, V.G. Pestov, Free topological groups over metrizable spaces, Topology Appl., 33(1989), 63-76.

[2] A. Arhangel'skiǔ, M. Tkachenko, Topological groups and related structures, Atlantis Press, Paris; World Scientific Publishing Co. Pte. Ltd., Hackensack, NJ, 2008.

[3] Z. Li, F. Lin, C. Liu, Networks on free topological groups, Topology Appl., 180 (2015), 186-198.

[4] F. Lin, C. Liu, $S_{\omega}$ and $S_{2}$ on free topological groups, Topology Appl., 176(2014), 10-21.

[5] F. Lin, C. Liu, The $k$-spaces property of the free Abelian topological groups over non-metrizable Lašnev spaces, Topology Appl., 220(2017), 31-42.

[6] A.A. Markov, On free topological groups, Amer. Math. Soc. Translation, 8 (1962), 195-272.

[7] V. V. Uspenskii, Free topological groups of metrizable spaces, Math. USSR Izv. 37(1991)657-680.

[8] K. Yamada, Characterizations of a metrizable space such that every $A_{n}(X)$ is a $k$-space, Topology Appl., 49(1993), 74-94.

[9] K. Yamada, Tightness of free abelian topological groups and of finite products of sequential fans, Topology Proc., 22(1997), 363-381.

[10] K. Yamada, Metrizable subspaces of free topological groups on metrizable spaces, Topology Proc., 23(1998), 379-409.

[11] K. Yamada, Fréchet-Urysohn spaces in free topological groups, Proc. Amer. Math. Soc., 130(2002), 2461-2469.

[12] K. Yamada, Fréchet-Urysohn subspaces of free topological groups, Topology Appl., 210(2016), 8189.

(Chuan Liu): Department of Mathematics, Ohio University Zanesville Campus, Zanesville, $\mathrm{OH}$ 43701, USA

E-mail address: liuc1@ohio.edu

(Fucai lin): School of mathematics and statistics, Minnan Normal University, Zhangzhou 363000, P. R. CHINA

E-mail address: linfucai2008@aliyun.com; linfucai@mnnu.edu.cn 